\title{
Development of a Novel Electromagnetic Double Action Meso-scale Pump
}

\author{
Ala'aldeen T. Al-Halhouli1,2, Nathir A. Rawashdeh ${ }^{1}$ \\ ${ }^{1}$ Department of Mechatronics Engineering \\ German Jordanian University \\ Amman, Jordan
}

\author{
Mousaa Sanna ${ }^{2}$, Stephanus Büttgenbach ${ }^{2}$, Andreas Dietzel ${ }^{2}$ \\ ${ }^{2}$ Institute of Microtechnology \\ Technische Universität Braunschweig \\ Braunschweig, Germany
}

\begin{abstract}
This paper presents the design and performance evaluation of a meso-scale electromechanical fluid pump. A prototype was designed and fabricated using Polycarbonate for the housings and controlled by sequential pulsing of channel piston and valve coils. The pumping concept is based on reciprocating a hard magnet acting as a piston in a circular channel, and synchronizing this movement with two electromagnetically actuated valves located at the pump inlet and outlet ports. The pumping system is programmed to allow simultaneous energization of a set of coils that control the magnet positions. Each pump port has an inlet and outlet channel. According to the piston movement direction, i. e. clockwise or counter clockwise, the valve will change its position to allow for inflow or outflow. The pump concept was tested on a meso-scale setup for pumping water. Tests showed that a magnet rotational speed of 70 strokes per minute is achievable. A flow rate of 6.1 $\mathrm{ml} / \mathrm{min}$ and a pressure of $400 \mathrm{~Pa}$ were obtained at this speed.
\end{abstract}

Keywords-electromagnetic; meso; pump; fluidics

\section{INTRODUCTION}

Pumps are the heart of hydraulic systems. When it comes to miniaturization of fluidic systems towards biomedical and lab-on-a-chip platforms, integrating meso/micro-scale pumps becomes the most challenging task in terms of functionality, flow rate control and pressure range. In the last decade, extensive work on microactuators, i.e. thermomechanical, piezo-electric, shape memory alloys, and magnetic has been published. This paves the way for numerous novel applications and became a key driving element in micropositioning, microhandling systems and in microfluidic devices [1].

Due to their considerable advantages, such as large actuation forces, large deflections, low driving voltages, fast response time, and robustness under harsh conditions, magnetic microactuators are very attractive $[2,3]$; however, their fabrication process is technologically challenging. Nevertheless, pumps employing UV depth lithography using new types of photoresists [4], micro-electroplating techniques and micromachined polymer magnets, lead to novel electromagnetically actuated microsystems [5-7] with promising applications in active microfluidic devices such as gear [6], centrifugal [7], spiral [8] and synchronous micropumps [9]. All these configurations require high magnetic fields to run the pumps.

The micro gear pump in [6] has two combined synchronous motors and is enclosed by an SU-8 chamber wall. Teeth with an involute shape are placed on the outer diameter of the rotors forming gears. One gear driven by the threephase system actuates the other gear which rotates in the opposite direction. While rotating, the fluid is drawn from the inlet, split to both gears and transported in the space between the teeth and the chamber wall. Both fluid streams are combined and displaced to the outlet. First tests showed successful tests with maximum rotational speeds up to $1000 \mathrm{rpm}$. A pump rate of $150 \mu \mathrm{l} / \mathrm{min}$ was obtained for one pump type at a rotational speed of $150 \mathrm{rpm}$ [6]

As for the centrifugal force pump in [7], the rotor of the synchronous micro-motor was modified to take a toothed disc shape. The rotor was mounted onto the stator and adjusted by an integrated guide. The toothed rotor was made of alternate magnets, which were realized by using polymer magnets fabrication technology. Tests showed that when the chamber was filled with fluid, a current of $100 \mathrm{~mA}$ was required for continuous rotor rotation compared to tests without a fluid. The pump was successfully driven over a long period of time with variable rotating speeds up to $4000 \mathrm{rpm}$. No flow rate measurements have been reported by this study.

The same driving mechanism was also tested to run a spiral channel viscous micropump that consists of a rotational spiral channel-disk, a flat cover, fluid inlet and outlet ports and a driving motor [8]. The inlet and outlet ports are located at either ends of the spiral channel. The spiral channel disk is driven by the motor and spins in close proximity to the flat cover. Consequently, the dominant viscous drag force between the rotational disk and the working fluid forces the flow to be in a direction tangential to the element's motion. The experimental parameters are the spiral channel disk speed and the pressure at the outlet port, i.e. back pressure. The speed ranges between 3000 and $4500 \mathrm{rpm}$. The maximum flow rate of $1023 \mu 1 / \mathrm{min}$ was achieved at a motor rotational speed of $4500 \mathrm{rpm}$ corresponding to an applied current of $50 \mathrm{~mA}$.

Interestingly, some research efforts were also directed toward using ferrofluidic plugs to create valve-less pumping 
based on a magnetic force $[10,11]$. The ferrofluidic plug is driven in an annular channel by an external magnet or by a series of actuating solenoids [11]. The ferrofluidic plugs pump is advantageous because the pumping action results from piston motion in the wafer plane, which allows a higher stroke volume and compression ratio without compromising power and voltage requirement of the driver. Pump dosing correlates highly with the velocity of the translating plug. The pump provides advantages such as: absence of valves, self priming, bubble tolerance. This gives it the potential of successful application in the highly demanding biological and biomedical microsystems; however, degradation of the ferrofluid in the annular channel is a drwaback.

To overcome the degradation disadvantage, a synchronous micropump based on microfabricated copper coils and polymer magnets was developed [9]. The pump works by synchronizing the rotation of two polymer magnets in a circular SU-8 microfluidic channel. Magnet rotation is achieved by sequentially activating a set of planar coils to repel or attract the travelling magnet through the channel, while the second one is anchored between the inlet and the outlet ports. At the end of each pumping cycle, the magnets exchange their anchored and travelling functions. The micropump has been successfully tested and water flow rates of $17.3 \mu \mathrm{l} / \mathrm{min}$ at $31.25 \mathrm{rpm}$ to $158.7 \mu \mathrm{l} / \mathrm{min}$ at $83.33 \mathrm{rpm}$ were achieved. Driven by these experimental results, the concept of synchronized double action pump was proposed. The main reason for that was the dominant dependence of the flow rates and operating pressures in the synchronous pumping concept on the timing, where magnets exchange their roles, and the anchoring force, which depends on the applied coil magnetic force, the repel force of the moving magnet and the fluid properties.

To avoid these issues, this study proposed a novel circular double action pumping design in the meso-scale. The pump has been developed and tested under different stroke frequencies. This design is down-scalable and is intended for microfabrication processing.

\section{MESO-SCALE PUMP DESIGN}

The meso-scale double action pump consists of a cover plate, three permanent magnets and pump housing. Fluidic channels, and solenoid grooves are made using precision machining techniques, $\mathrm{CNC}$ milling machine, in Polycarbonate. Polycarbonate is a synthetic plastic of the polyester family. It is an amorphous material with a crystalline proportion of less than 5\%. Polycarbonates are characterized by high hardness and rigidity. In addition, they are good dielectrics and transparent, but can be tinted using various color dies. They are resistant to water, several mineral acids, and water-based solutions of neutral salts and oxidation agents. The pump housing consists of a circular channel in which a permanent magnet is actuated electromagnetically via coils to act as a piston pushing the fluid inside the channel. Two inlets and two outlets are drilled into the Polycarbonate pump cover and are opened and closed by two further valve magnets. The movement of the piston magnet in the clockwise and counter-clockwise directions is coordinated with the movement of the valve magnets to achieve the pumping of fluid through the two inlets and two outlets. The conceptual design and operation are illustrated in Fig. 1 and Fig. 2. The direction of the piston and valve magnet movement velocity can be controlled by changing the direction and frequency of current pulses in the coil windings wrapped around the fluidic channel.

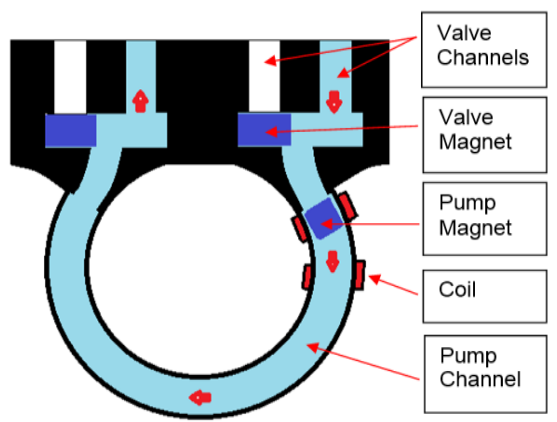

Fig. 1. Pump concept in forward stroke.

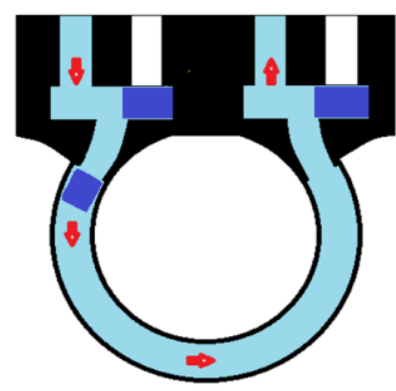

Fig. 2. Pump concept in backward stroke.

In the electromechanical pump three permanent magnets act as inlet/outlet valves and a piston that pushes the fluid through the circular channel. The latter is achieved by actuating the channel coils in sequence while controlling the current pulse frequency and direction. The two valve magnets are placed in opposing polarity to prevent them from sticking to each other, given that the valves are in close proximity. The valve magnets are moved once for every stroke to facilitate the passing of pumped fluid through the inlets and outlets as illustrated in Fig. 3.
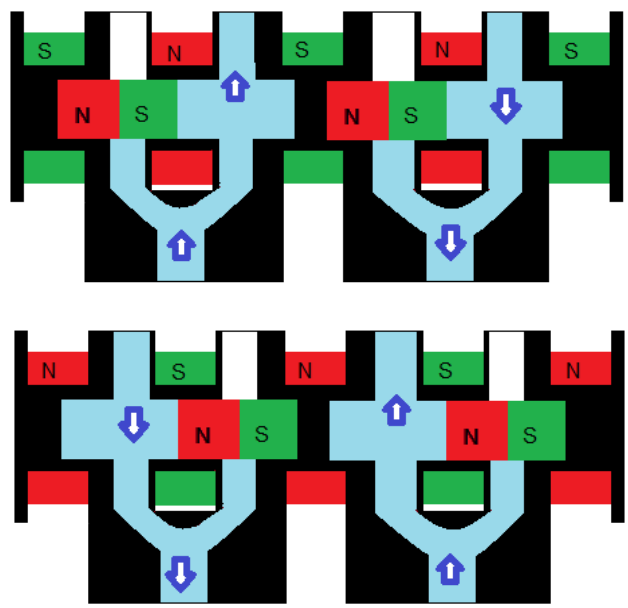

Fig. 3. Valving concept in backward and forward strokes. 
The pump prototype with attached inlet and outlet hoses, as well as actuation coils can be seen in Fig. 4. It shows a fluid channel of $(2 \times 2 \mathrm{~mm} 2+0.1 \mathrm{~mm}$ tolerance $)$ at an inner diameter of $16 \mathrm{~mm}$, and an outer diameter of $18 \mathrm{~mm}$. The pump cover has 4 drilled inlet and outlet ports. Three block $\mathrm{NdFeB}$ permanent magnets (material grade N38) from Chen Yang Technologies $\mathrm{GmbH}$ are used as valves and a piston. The reciprocating piston magnet is of $2 \times 2 \times 3 \mathrm{~mm}^{3}$ dimensions was set in the circular while two valving $2 \times 2 \times 6$ $\mathrm{mm}^{3}$ magnets were set in the valve magnet channel in opposing polarity. The cover is then fixed to the pump housing. Using block magnets enables verifying the pumping concept; however, due to increase in leakage rate through the magnet, lower flow rates and back pressures are expected. For the solenoids, $250 \mathrm{~mm}$ copper wire was wrapped in 230 turns around the fluidic channel with a total of $5 \Omega$ resistance per coil. The inlet and outlet hoses are connected to the cover holes. The pump prototype consist of ten piston moving coils on the circular channel, and five valve coils.

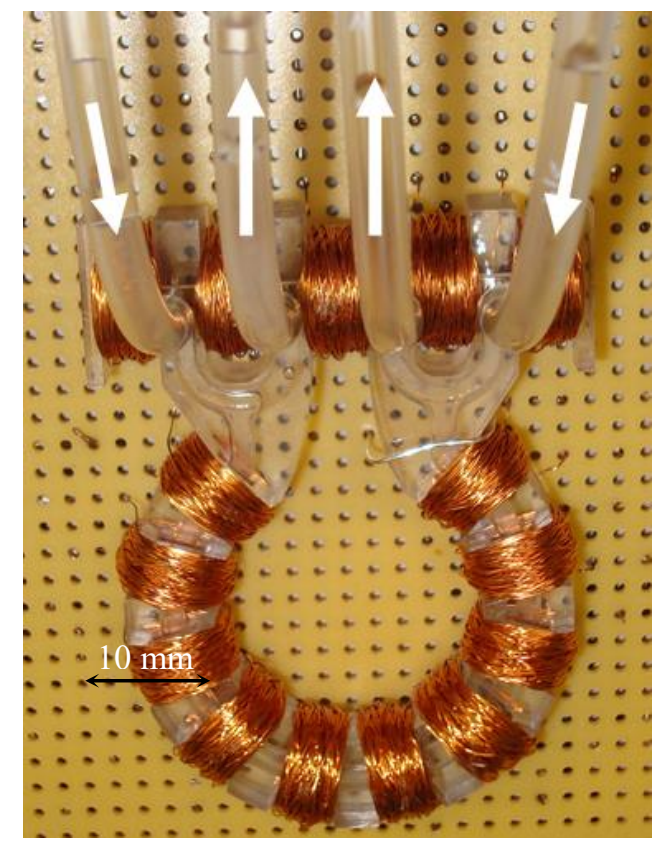

Fig. 4. Micro-pump prototype with connected fluid in- and outlets.

\section{EXPERIMENTAL RESULTS}

Several experiments were conducted in order to measure the performance of the pump. Measured quantities include: volume flow rate $Q$ in milliliter per minute $(\mathrm{ml} / \mathrm{min})$; maximum fluid height in millimeter $(\mathrm{mm})$; the fluid pressure in Pascal $(\mathrm{Pa})$. Input variables include: the stroke frequency in strokes per minute (spm); the fluid pressure difference in Pascal; the outlet height in millimeter.

A micro controller was used to synchronize the coil currents, but required an external triggered power source because the microcontroller cannot source the amount of current necessary to move the permanent magnets inside the pump. Initial tests of proposed energizing schemes were run first on a DUO-LED pump simulator shown in Fig. 5.

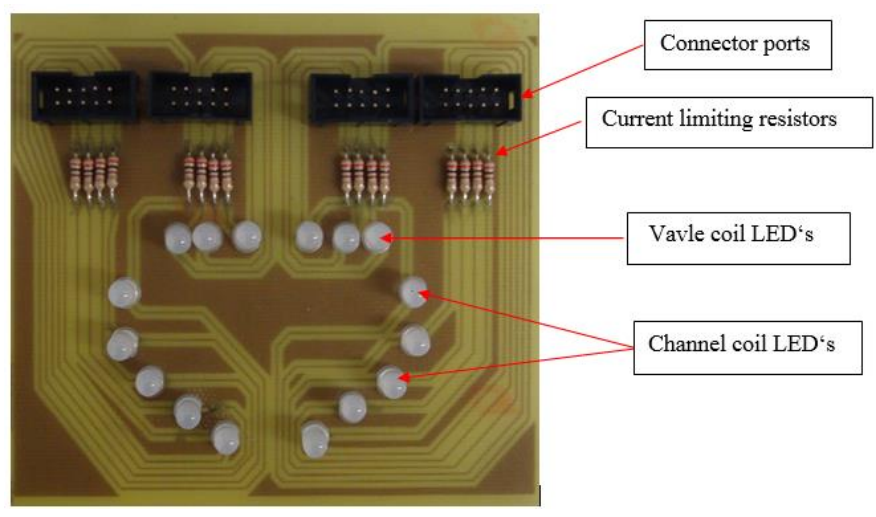

Fig. 5. DUO-LED pump simulator circuit board.
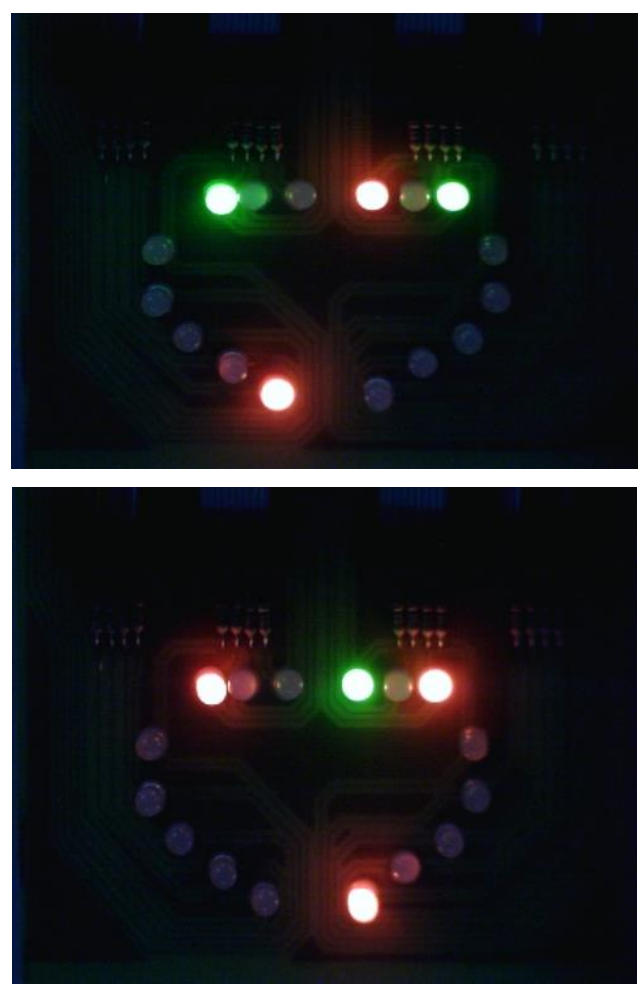

Fig. 6. DUO-LED pump simulator tests.

Two samples of tests on simulator are shown in Figure 6. Simulator verified schemes are then run on the pump itself and the amount of pumped fluid was measured using an electrical scale. To achieve more accurate results, flow rates were averaged over five readings per test configuration.

In the experiments, two coils were energized at a time to move the piston magnet. The piston magnet speed is varied by inserting a variable coil energizing delay between 650 and 200 milliseconds. An unforeseen interference between the valve magnets and coils with the piston magnet, forced the experiment to be conducted with the piston magnet moving between the coils 3 and 9 rather than 1 and 10 . The experimental results shown in Fig. 7 through Fig. 9 validate the pump design concept and point to linear trends, which simplify the prediction of pump performance for a given application. 


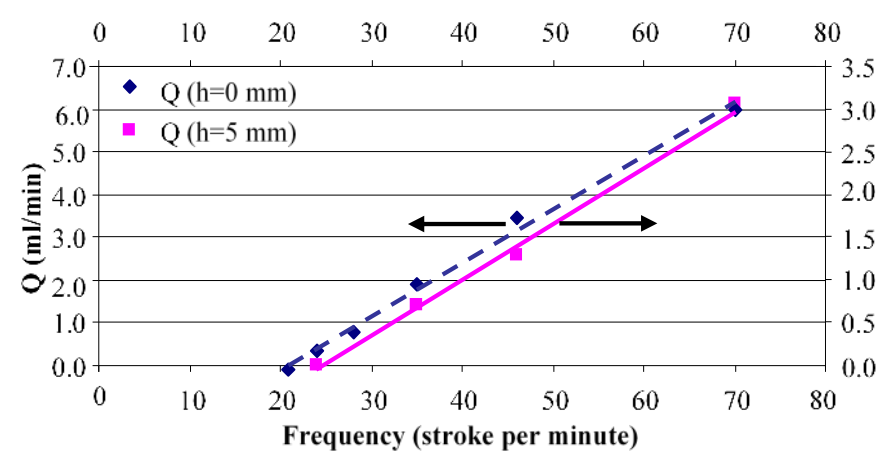

Fig. 7. Flow rate as a function of operating frequency and outlet height.

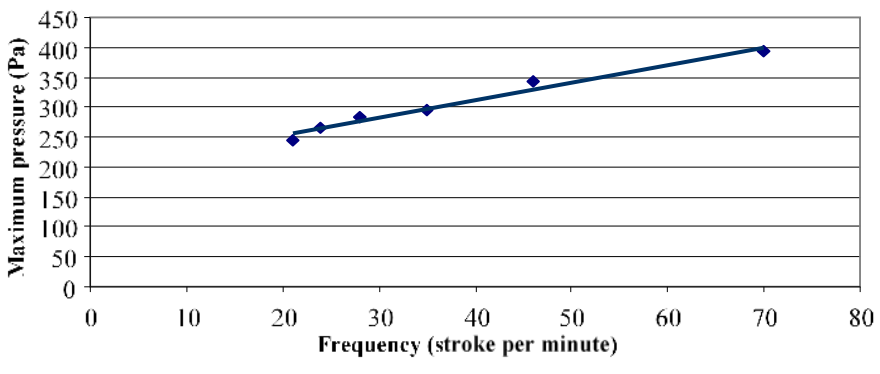

Fig. 8. Maximum pressure difference as a function of operating frequency.

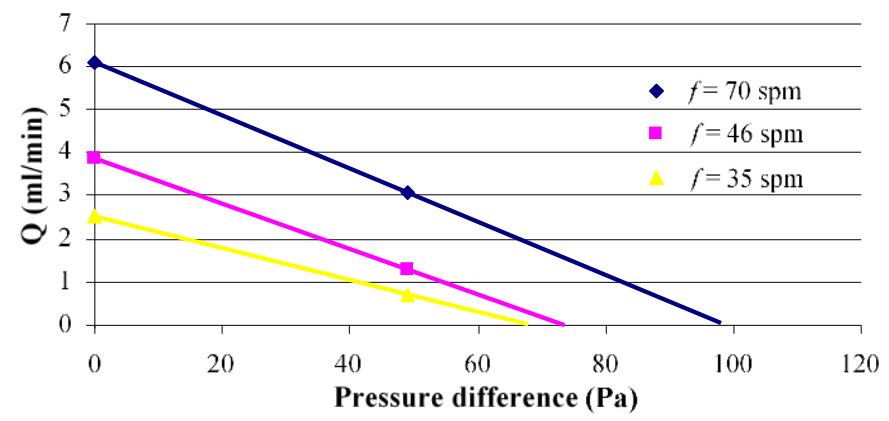

Fig. 9. Volume flow rate as a function pressure difference for different operating frequencies in stokes/min.

For the tested pump frequency range, results showed that the flow rate and maximum pressures are increasing nearly linearly with pump frequency and decreasing linearly with increasing pressure difference. Using these figures and plotting the linear trend lines of the flow rates with pressure at different rotational speeds provide us with the maximum pressure difference and flow rates expected values. In case of $70 \mathrm{spm}$, it is estimated to be $400 \mathrm{~Pa}$ and $6.1 \mathrm{ml} / \mathrm{min}$ respectively.

In order to compare the presented pump with other miniaturized pumps, a list of previously developed water pumps and their characteristics are summarized in Table 1.
TABLE I. COMPARISON OF ELECTROMAGNETIC PUMP CONCEPTS.

\begin{tabular}{|c|l|c|c|c|c|c|}
\hline Ref. & \multicolumn{1}{|c|}{ Pump Type } & W & H & D & Freq. & $\mathbf{Q}_{\max }$ \\
\hline$[6]$ & Gear & - & 0.8 & $7.5-10$ & 150 & 0.150 \\
\hline$[8]$ & Spiral & - & - & 4.5 & 4500 & 1.023 \\
\hline$[9]$ & $\begin{array}{l}\text { Synchronous- } \\
\text { Microscale }\end{array}$ & 1.5 & 0.8 & - & 83.33 & 0.158 \\
\hline$[10]$ & Ferrofluidic & 0.25 & 2 & - & 8 & 0.0478 \\
\hline$[12]$ & $\begin{array}{l}\text { Synchronous-Meso- } \\
\text { scale }\end{array}$ & 2 & 2 & - & 200 & 13.7 \\
\hline self & $\begin{array}{l}\text { Double-action-Meso- } \\
\text { scale }\end{array}$ & 2 & 2 & - & 70 spm & 6.1 \\
\hline
\end{tabular}

\section{CONCLUSIONS}

A novel electromagnetic actuation based double action pumping concept that depends on simultaneous energization of three permanent magnets placed in a circular and valving channels has been realized and tested.

In the electromechanical pump three permanent magnets act as inlet/outlet valves and a piston that pushes the fluid through the circular channel. The two valve magnets are placed in opposing polarity to prevent them from sticking to each other, given that the valves are in close proximity. The valve magnets are moved once for every stroke to facilitate the passing of pumped fluid through the inlets and outlets.

A maximum flow rate of $6.1 \mathrm{ml} / \mathrm{min}$ and a pressure of 400 $\mathrm{Pa}$ at $70 \mathrm{spm}$ were achieved. Pump components and their fabrication are suitable for realization in microscales and are under consideration. Further investigations on the energization schemes, coil design and fluid leakage between the permanent magnets and wall channels are highly recommended.

It is expected that downscaling the pump components dimensions (foot print of $\approx 1 \mathrm{~cm}$ ) is possible with the well established technology available at IMT. The pump will offer controllable low flow rates and could work against relatively high back pressures. It could be also as an integrated active device as a driver for microfluidic platforms.

\section{ACKNOWLEDEGMENTS}

The authors gratefully acknowledge the support of the German Research Foundation (DFG) in the framework of the Collaborative Research Group mikroPART (Microsystems for particulate life science products). The first author (A. AlHalhouli) thanks the German Research Foundation (DFG) for financial support of a three-month stay at Technische Universität Braunschweig. 


\section{REFERENCES}

[1] S. Büttgenbach, A. Burisch, J. Hesselbach (Eds.), "Design and manufacturing of active microsystems", Springer, Dordrecht, 2011.

[2] I.J. Busch-Vishniac, "The case for magnetically driven microactuators", Sensors and Actuators, A. 33, 1992, pp. 207-220.

[3] O. Cugat, J. Delamare, G. Reyne, "Magnetic micro-actuators and systems (MAGMAS)", IEEE Transactions on Magnetics, 39, 2003, pp. 3607-3612.

[4] H. Lorenz, M. Despont, N. Fahrni, J. Brugger, P. Vettiger, P. Renaud, "High-aspect-ratio, ultrathick, negative-tone near-UVB photoresist and its application for MEMS", Sensors and Actuators, A. 64, 1998, pp. 3339.

[5] A. T. Al-Halhouli, "Recent advances in on-disk viscous micropumps", Journal of Microelectronics and Electronic Packaging, 6, 2009, pp. 1-9.

[6] A. Waldschik, S. Büttgenbach, "Micro gear pump with internal electromagnetic drive", Microsystem Technology, 16, 2010, pp. 15811587.

[7] A. Waldschik, S. Büttgenbach, "Fabrication of internally driven micro centrifugal force pumps based on synchronous micro motors", Microsystem Technology, 16, 2010, pp. 1105-1110.

[8] A. T. Al-Halhouli, S. Demming, A. Waldschik, S. Büttgenbach, "Implementation of synchronous micromotor in developing integrated microfluidic systems", Micromachines, Micromachines, 2014, 5(3), pp. $442-456$.

[9] A.T. Al-Halhouli, M. I. Kilani, A. Waldschik, A. Phataralaoha, S. Büttgenbach, "Development and testing of a synchronous micropump based on electroplated coils and microfabricated polymer magnets", Journal of Micromechanics Microengineering, 22, 2012.

[10] A. Hatch, A. E. Kamholz, G. Holman, P. Yager, K. F. Bohringer, “A ferrofluidic magnetic micropump", Journal of Microelectromechanical Systems, 2001, 10, pp. 215.

[11]N. Nguyen, M. Chai, "A stepper micropump for ferrofluid driven microfluidic systems", Micro and Nanosystems, 2009,1, pp. 17.

[12] A.T. Al-Halhouli, M. I. Kilani, and S. Büttgenbach, "Development of a Novel Electromagnetic Pump for Bio-medical Applications", Sensors and Actuators A, 2010, 162 (2), pp. 172-176. 